\title{
MYD88 L265P mutation analysis helps define nodal lymphoplasmacytic lymphoma
}

\author{
Fatima Hamadeh ${ }^{1}$, Stephen P MacNamara ${ }^{1}$, Nadine S Aguilera ${ }^{2}$, Steven H Swerdlow ${ }^{3}$ and \\ James R Cook ${ }^{1}$ \\ ${ }^{1}$ Department of Laboratory Medicine, Cleveland Clinic, Cleveland, OH, USA; ${ }^{2}$ Department of Pathology, \\ University of Virginia, Charlottesville, VA, USA and ${ }^{3}$ Division of Hematopathology, University of Pittsburgh, \\ Pittsburgh, PA, USA
}

\begin{abstract}
The diagnosis of lymphoplasmacytic lymphoma is often challenging, especially in extramedullary tissues where the differential diagnosis includes nodal marginal zone lymphoma, splenic marginal zone lymphoma, or other small B-cell neoplasms with plasmacytic differentiation. The MYD88 L265P mutation has been recently identified in $>90 \%$ of bone-marrow-based lymphoplasmacytic lymphoma, but the incidence of this abnormality and corresponding morphologic correlates in nodal lymphoplasmacytic lymphoma have not been established. We analyzed 87 cases of extramedullary lymphoplasmacytic lymphoma, splenic marginal zone lymphoma, unclassifiable splenic B-cell lymphomas, nodal marginal zone lymphoma with plasmacytic differentiation, and chronic lymphocytic leukemia/small lymphocytic lymphoma with plasmacytic differentiation for MYD88 L265P. Eighteen cases $(21 \%)$ were positive, including 9/9 (100\%) lymphoplasmacytic lymphomas with classic histologic features, 5/12 (42\%) cases that met 2008 WHO criteria for lymphoplasmacytic lymphoma but with atypical morphologic features, $3 / 15(20 \%)$ cases initially considered nodal marginal zone lymphoma with plasmacytic differentiation, and 1/6 (17\%) unclassifiable splenic B-cell lymphomas. The presence of MYD88 L265P was associated with IgM paraprotein $(P<0.001)$ and a trend for bone marrow involvement $(P=0.09)$. Each of 44 splenectomy-defined splenic marginal zone lymphomas (19 with plasmacytic differentiation) and the chronic lymphocytic leukemia/small lymphocytic lymphoma with plasmacytic differentiation were negative for the mutation. Morphologic re-review with knowledge of MYD88 mutation status and all available clinical features suggested all MYD88 mutated cases were consistent with lymphoplasmacytic lymphoma (either classic or variant histology), except for one case which remained most consistent with nodal marginal zone lymphoma with plasmacytic differentiation. These results demonstrate the importance of MYD88 mutational analysis in better defining lymphoplasmacytic lymphoma as a relatively monomorphic small B-cell lymphoma with plasmacytic differentiation that may show total nodal architectural effacement and follicular colonization. Cases previously considered lymphoplasmacytic lymphoma that are more polymorphous and are often associated with histiocytes should no longer be included in the lymphoplasmacytic lymphoma category. Clinicopathologic review suggests that although MYD88 mutated non-lymphoplasmacytic lymphoma small B-cell neoplasms exist, they are very uncommon.
\end{abstract}

Modern Pathology (2015) 28, 564-574; doi:10.1038/modpathol.2014.120; published online 12 September 2014

Although lymphoplasmacytic lymphoma/Waldenstrom's macroglobulinemia has been recognized as a clinical entity for decades, diagnostic criteria for lymphoplasmacytic lymphoma have always been controversial. ${ }^{1-6}$ The current WHO classification defines lymphoplasmacytic lymphoma as a B-cell lymphoid neoplasm composed of small B lympho-

Correspondence: Dr JR Cook MD, PhD, Department of Laboratory Medicine, Cleveland Clinic, 9500 Euclid Ave., Cleveland, OH 44195, USA.

E-mail: cookj2@ccf.org

Received 17 June 2014; accepted 30 July 2014; published online 12 September 2014 cytes, plasmacytoid lymphocytes, and plasma cells, usually involving bone marrow and sometimes lymph nodes and spleen, which does not fulfill the criteria for any of the other small B-cell lymphoid neoplasms. ${ }^{2}$ The diagnosis of lymphoplasmacytic lymphoma is therefore in large part a diagnosis of exclusion. As most cases of lymphoplasmacytic lymphoma are diagnosed from bone marrow biopsies, establishing a diagnosis of lymphoplasmacytic lymphoma from lymph node or spleen can be particularly challenging.

We and others have previously shown that nodal small B-cell lymphomas meeting WHO criteria as lymphoplasmacytic lymphoma are morphologically heterogeneous. ${ }^{4,7-10}$ Prototypical cases exhibit partial 
architectural preservation with prominently dilated sinuses and a relatively monomorphic lymphoplasmacytic infiltrate, often with hemosiderin deposition and increased mast cells, without prominent follicular colonization. Cases with these features are usually associated with bone marrow involvement and an IgM paraprotein (ie, Waldenstrom's macroglobulinemia). ${ }^{7,10}$ Other cases, however, are more heterogeneous with effacement of the lymph node sinuses, a sometimes vaguely nodular appearance, and/or a more polymorphic infiltrate that includes a variable proportion of large transformed cells and/or histiocytes. These more heterogeneous cases have been shown to be less frequently associated with an IgM paraprotein and to be less frequently associated with bone marrow involvement. ${ }^{7,10}$ In light of these morphologic differences, it has been unclear whether all cases meeting current WHO criteria as lymphoplasmacytic lymphoma truly represent one neoplasm.

Recently, next generation sequencing studies have identified a highly recurrent point mutation, MYD88 L265P, in $>90 \%$ of cases of bone-marrow-based lymphoplasmacytic lymphoma/Waldenstrom's macroglobulinemia, ${ }^{11-16}$ suggesting that this mutation may serve as a sensitive diagnostic marker for lymphoplasmacytic lymphoma. A previous study has suggested that detection of the MYD88 L265P abnormality can assist in classifying challenging bonemarrow-based B-cell neoplasms with plasmacytic differentiation. ${ }^{17}$ In the current study, we have examined 87 cases of nodal or splenic-based B-cell lymphomas to identify the morphologic correlates of MYD88 L265P mutation in this setting and determine whether molecular studies for this abnormality can assist in classifying nodal and splenic lymphomas with plasmacytic differentiation in routine practice.

\section{Materials and methods}

\section{Case Selection and Morphologic Review}

This study was approved by the Cleveland Clinic and University of Pittsburgh Institutional Review Boards. The authors' files were searched for lymph nodes or spleens diagnosed as lymphoplasmacytic lymphoma, splenic marginal zone lymphoma or unclassifiable splenic lymphoma (with or without plasmacytic differentiation), or other small B-cell neoplasms with plasmacytic differentiation. Cases without residual archived material for MYD88 mutation analysis were excluded. A total of 87 cases were identified.

Morphologic review was performed in two stages. Initial pathologic review (FH and JRC) was performed using 2008 WHO criteria $^{18}$ without knowledge of MYD88 L265P mutation status but with any available clinical data, routine hematoxylin and eosin-stained sections, and data from corresponding flow cytometric, immunophenotypic, and/or cytogenetic studies. Following MYD88 mutation analysis, all atypical lymphoplasmacytic lymphoma cases and all non-lymphoplasmacytic lymphoma cases with MYD88 mutation were re-reviewed (JRC and SHS) with all clinical and laboratory data from the initial review as well as knowledge of MYD88 mutation status and, where available, corresponding bone marrow findings.

\section{MYD88 L265P Mutation Analysis}

DNA was isolated from formalin-fixed, paraffinembedded tissue blocks or unstained slides and PCR for MYD88 L265P was performed using a realtime assay (qBiomarker somatic mutation assay for MYD88_85940, Qiagen, Valencia, CA, USA) according to the manufacturer's instructions on the LightCycler 480 (Roche, Indianapolis, IN, USA). This assay employs two separate real-time reactions: one specific for the MYD88 L265P mutation and one that targets a reference, non-mutated portion of the MYD88 gene. A crossing point $\left(\mathrm{C}_{\mathrm{T}}\right)$ value is calculated for the mutant and reference MYD88 reactions, and a $\Delta \mathrm{C}_{\mathrm{T}}$ is computed $\left(\Delta \mathrm{C}_{\mathrm{T}}=\mathrm{C}_{\mathrm{T}}\right.$ mutant_$\mathrm{C}_{\mathrm{T}}$ reference). Analysis of 120 normal peripheral blood samples identified a cutoff of $\Delta \mathrm{C}_{\mathrm{T}}<10$ for interpretation as positive for the MYD88 L265P mutation. Serial dilution studies using the mutation-positive OCI-LY10 and mutation-negative OCI-LY19 cell lines demonstrated a limit of detection of $0.5 \%$ mutant alleles (1\% heterozygous tumor cells).

\section{Statistics}

Statistical analysis was performed using GraphPad Prism 6 software (GraphPad software, San Diego, CA, USA). Categorical variables were compared using a two-tailed Fisher exact test for $2 \times 2$ contingency tables or Chi-square analysis for larger tables. Continuous variables were compared using Student's $t$-test.

\section{Results}

\section{Initial Morphologic Review}

Each of the 87 cases was reviewed without knowledge of MYD88 mutation status and classified using the 2008 WHO criteria. ${ }^{18}$ There were 9 classic lymphoplasmacytic lymphoma with preservation of sinuses and a relatively monomorphic small lymphoplasmacytic infiltrate (Figure 1), 12 atypical lymphoplasmacytic lymphomas that were designated for the purposes of this study as small B-cell neoplasm with plasmacytic differentiation, not otherwise specified, 15 nodal marginal zone lymphoma with plasmacytic differentiation, 44 splenic marginal zone lymphomas including 19 with plasmacytic differentiation, 6 unclassifiable splenic B-cell lymphomas including 3 with plasmacytic differentiation, and 1 chronic lymphocytic leukemia/small lymphocytic lymphoma with plasmacytic differentiation 

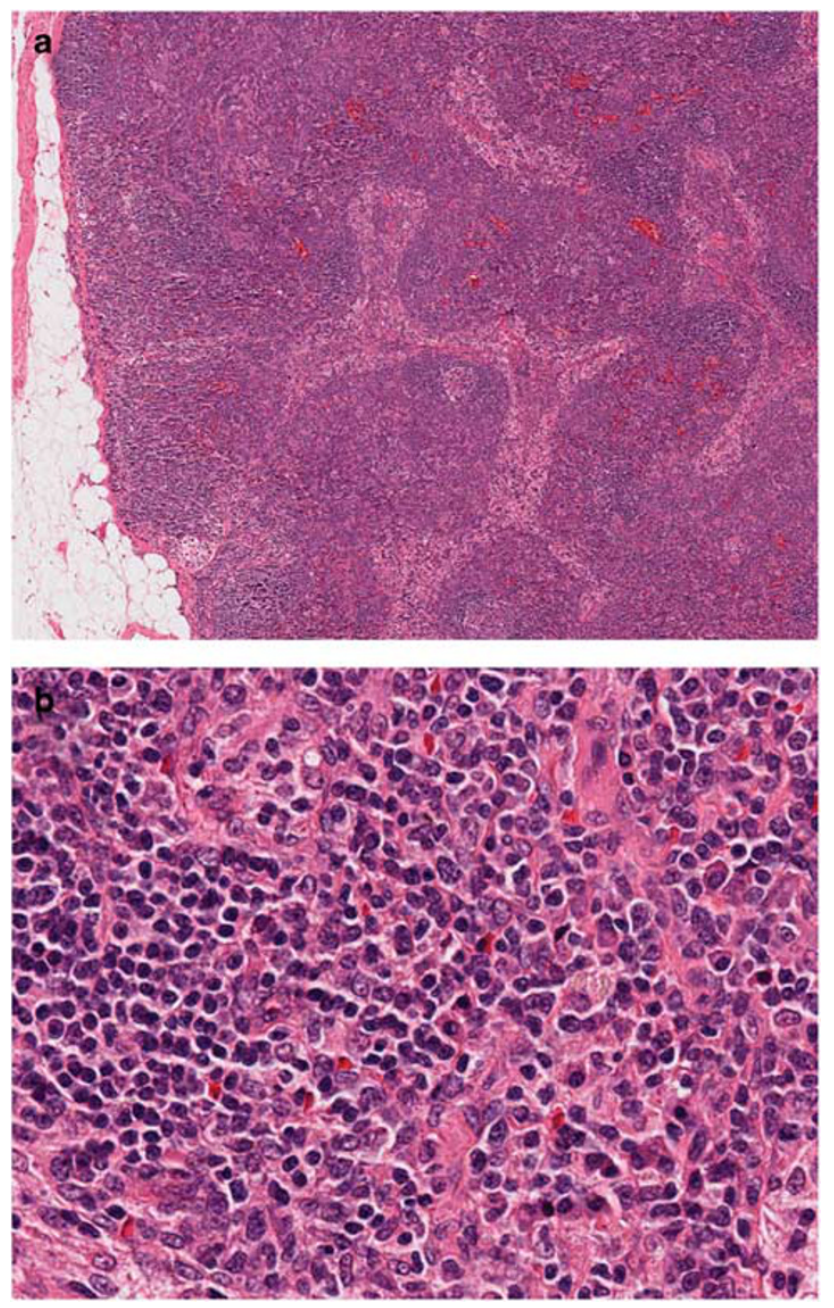

Figure 1 Classic lymphoplasmacytic lymphoma morphology was defined by architectural preservation with patent sinuses (a) and a relatively monomorphic infiltrate of small lymphocytes and plasma cells (b).

involving a lymph node. The clinical, laboratory, phenotypic, and molecular findings in each category are summarized in Table 1.

\section{MYD88 L265P Mutation Analysis}

Allele-specific PCR for the MYD88 L265P mutation was performed from formalin-fixed, paraffin-embedded tissues (Table 1). Eighteen cases $(21 \%)$ were positive, including each of the 9 cases of classic lymphoplasmacytic lymphoma (100\%), 5/12 small B-cell neoplasms with plasmacytic differentiation, not otherwise specified ( $42 \%$ ), 3/15 nodal marginal zone lymphomas with plasmacytic differentiation $(20 \%)$, and 1/6 unclassifiable splenic B-cell lymphomas (17\%). None of the 44 cases of splenic marginal zone lymphomas was positive for the mutation. Positivity for MYD88 L265P was associated with the presence of an IgM paraprotein
$(P<0.001)$ and a trend towards bone marrow involvement $(P=0.09)$, but not with age, gender, or light chain usage (Table 2).

\section{Morphologic Re-review in Light of MYD88 Status}

Next, all cases of small B-cell neoplasm with plasmacytic differentiation, not otherwise specified, and all other non-lymphoplasmacytic lymphomas with MYD88 mutation (three originally classified as nodal marginal zone lymphoma with plasmacytic differentiation and one unclassifiable splenic B-cell lymphoma), were re-reviewed with knowledge of MYD88 mutation status. Three of the MYD88 mutated cases initially classified as small B-cell neoplasm with plasmacytic differentiation, not otherwise specified, showed similar features with diffuse effacement of the lymph node architecture and extracapsular infiltration by a relatively monomorphic lymphoplasmacytic infiltrate similar to that seen in classic lymphoplasmacytic lymphoma (Figure 2). Each of these three cases was associated with an IgM monoclonal protein and bone marrow involvement. These cases were reclassified as lymphoplasmacytic lymphoma showing complete nodal effacement.

Two MYD88 mutated small B-cell neoplasm with plasmacytic differentiation, not otherwise specified, cases and two MYD88 mutated cases initially classified as nodal marginal zone lymphomas with plasmacytic differentiation could also be grouped together as showing similar findings as defined by partial architectural preservation, a relatively monomorphic lymphoplasmacytic infiltrate, vague nodularity with variably prominent follicular colonization, and only focal areas with monocytoid appearance (Figure 3). Of these four cases, the two with relatively more prominent follicular colonization had initially been classified as nodal marginal zone lymphoma with plasmacytic differentiation, whereas the two with less prominent but still apparent nodularity had been classified as small B-cell neoplasm with plasmacytic differentiation, not otherwise specified. Each of these cases also showed bone marrow involvement and three were associated with an IgM paraprotein. In the remaining case, a serum electrophoresis study near the time of biopsy showed an IgG Kappa monoclonal protein, but another electrophoresis study 1 year prior had shown both IgG and IgM paraproteins and immunohistochemistry showed the lymphoma to be associated with IgM Kappa, not IgG-positive plasma cells. These cases were reclassified as lymphoplasmacytic lymphoma with a vaguely nodular appearance and follicular colonization.

One MYD88 mutated case initially considered an unclassifiable splenic B-cell lymphoma showed a marked red pulp infiltration of small lymphoid cells with little remaining white pulp (Figure $4 \mathrm{a}$ and b). Plasmacytic differentiation in this case consisted of 
Lymphoplasmacytic lymphoma, WHO (n=21)

\begin{tabular}{|c|c|c|c|c|c|c|c|}
\hline & \multirow[b]{2}{*}{ Total $(\mathrm{n}=87)$} & \multirow[b]{2}{*}{$\begin{array}{l}\text { Splenic marginal } \\
\text { zone lymphoma } \\
(\mathrm{n}=44)\end{array}$} & \multirow[b]{2}{*}{$\begin{array}{c}\text { Splenic B-cell } \\
\text { lymphoma, } \\
\text { unclassifiable }(\mathrm{n}=6)\end{array}$} & & \multirow[b]{2}{*}{$\begin{array}{c}\text { Nodal marginal } \\
\text { zone lymphoma } \\
\text { with plasmacytic } \\
\text { differentiation }(\mathrm{n}=15)\end{array}$} & \multirow[b]{2}{*}{$\begin{array}{c}\text { Chronic lymphocytic } \\
\text { leukemia/small lymphocytic } \\
\text { lymphoma with } \\
\text { plasmacytic } \\
\text { differentiation }(\mathrm{n}=1)\end{array}$} \\
\hline & & & & $\begin{array}{l}\text { Lymphoplasmacytic } \\
\text { lymphoma, } \\
\text { classic }(\mathrm{n}=9)\end{array}$ & $\begin{array}{c}\text { Small B-cell neoplasm } \\
\text { with plasmacytic } \\
\text { differentiation, } \\
\text { not otherwise } \\
\text { specified }(\mathrm{n}=12)\end{array}$ & & \\
\hline Male & $47(54 \%)$ & $25(57 \%)$ & $2(33 \%)$ & $6(67 \%)$ & $6(50 \%)$ & $7(47 \%)$ & $1(100 \%)$ \\
\hline Median age (range) & $64.5(44-89)$ & $65.5(45-89)$ & $66.5(54-80)$ & $57(47-79)$ & $60.5(44-82)$ & $66(49-84)$ & 70 \\
\hline Bone marrow involved & $41 / 55(75 \%)$ & $23 / 28(82 \%)$ & $2 / 2(100 \%)$ & $5 / 5(100 \%)$ & $7 / 10(70 \%)$ & $3 / 9(33 \%)$ & $1 / 1(100 \%)$ \\
\hline Bone marrow not involved & $14 / 55(25 \%)$ & $5 / 28(18 \%)$ & $0 / 2(0 \%)$ & $0 / 5(0 \%)$ & $3 / 10(30 \%)$ & $6 / 9(67 \%)$ & $0 / 1(0 \%)$ \\
\hline Bone marrow not available & 32 & 16 & 4 & 4 & 2 & 6 & 0 \\
\hline \multicolumn{8}{|c|}{ Serum protein electrophoresis monoclonal protein: } \\
\hline Present & $29 / 41(71 \%)$ & $8 / 15(53 \%)$ & $1 / 1(100 \%)^{\mathrm{a}}$ & $6 / 6(100 \%)$ & $8 / 9(89 \%)$ & $5 / 9(56 \%)$ & $1 / 1(100 \%)$ \\
\hline IgM & $20 / 40(50 \%)$ & $5 / 15(33 \%)$ & Not available & $6 / 6(100 \%)$ & $7 / 9(78 \%)$ & $2 / 9(22 \%)$ & $0 / 1(0 \%)$ \\
\hline IgG & $9 / 40(22 \%)$ & $3 / 15(20 \%)$ & Not available & $0 / 6(0 \%)$ & $1 / 9(11 \%)$ & $3 / 9(33 \%)$ & $1 / 1(100 \%)$ \\
\hline Absent & $12 / 40(30 \%)$ & $7 / 15(47 \%)$ & $0 / 1(0 \%)$ & $0 / 6(0 \%)$ & $1 / 9(11 \%)$ & $4 / 9(44 \%)$ & $0 / 1(0 \%)$ \\
\hline Not available & 46 & 29 & 5 & 3 & 3 & 6 & 0 \\
\hline \multicolumn{8}{|c|}{ Plasma cell immunohistochemistry: } \\
\hline N/A & 2 & 1 & 0 & 0 & 0 & 1 & 0 \\
\hline Polytypic & $28 / 85(33 \%)$ & $25 / 43(58 \%)$ & $3 / 6(50 \%)$ & $0 / 9(0 \%)$ & $0 / 12(0 \%)$ & $0 / 14(0 \%)$ & $0 / 1(0 \%)$ \\
\hline Monotypic & $57 / 85(67 \%)$ & $18 / 43(42 \%)$ & $3 / 6(50 \%)$ & $9 / 9(100 \%)$ & $12 / 12(100 \%)$ & $14 / 14(100 \%)$ & $1 / 1(100 \%)$ \\
\hline $\mathrm{K}$ & $46 / 57(81 \%)$ & $14 / 18(77 \%)$ & $1 / 3(33 \%)$ & $8 / 9(89 \%)$ & $9 / 12(75 \%)$ & $14 / 14(100 \%)$ & $0 / 1(0 \%)$ \\
\hline l & $11 / 57(19 \%)$ & $4 / 18(22 \%)$ & $2 / 3(67 \%)$ & $1 / 9(11 \%)$ & $3 / 12(25 \%)$ & $0 / 14(0 \%)$ & $1 / 1(100 \%)$ \\
\hline $\operatorname{IgM}$ & $43 / 51(84 \%)$ & $11 / 13(85 \%)$ & $3 / 3(100 \%)$ & $9 / 9(100 \%)$ & $9 / 12(75 \%)$ & $11 / 14(79 \%)$ & N/A \\
\hline IgG & $7 / 51(14 \%)$ & $2 / 13(15 \%)$ & $0 / 3(0 \%)$ & $0 / 9(0 \%)$ & $3 / 12(25 \%)$ & $2 / 14(14 \%)$ & N/A \\
\hline IgA & $1 / 51(2 \%)$ & $0 / 13(0 \%)$ & $0 / 3(0 \%)$ & $0 / 9(0 \%)$ & $0 / 12(0 \%)$ & $1 / 14(7 \%)$ & N/A \\
\hline Heavy chain N/A & 6 & 5 & 0 & 0 & 0 & 0 & 1 \\
\hline MYD88 L265P positive & $18(21 \%)$ & $0(0 \%)$ & $1(17 \%)$ & $9(100 \%)$ & $5(42 \%)$ & $3(20 \%)$ & $0(0 \%)$ \\
\hline
\end{tabular}

${ }^{\mathrm{a}} \mathrm{M}$ protein present by electrophoresis but immunofixation not performed. 
Table 2 Correlation of MYD88 mutation status with clinical, laboratory, and phenotypic features

\begin{tabular}{lccr}
\hline & $\begin{array}{c}\text { MYD88 mutant } \\
\mathrm{n}=18\end{array}$ & $\begin{array}{c}\text { MYD88 wild type } \\
\mathrm{n}=69\end{array}$ & $\mathrm{P}$ \\
\hline Age & $59(47-80)$ & $66(44-89)$ & 0.12 \\
Male & $12(67 \%)$ & $35(51 \%)$ & 0.3 \\
Bone marrow & & & \\
Positive & 9 & 28 & 0.09 \\
Negative & 0 & 13 & \\
NA & 9 & 28 & \\
M protein & & & \\
IgM & 12 & 8 & \\
IgG & 1 & 7 & \\
Neg & 0 & 42 & \\
NA & 5 & & \\
Light chain IHC & & 31 & \\
Kappa & 15 & 8 & \\
Lambda & 3 & 2 & \\
NA & 0 & & \\
& & &
\end{tabular}

a minor population of IgM Lambda plasma cells. A bone marrow biopsy in this case showed diffuse infiltration by small lymphocytes and lambda monotypic plasma cells, without an overt intrasinusoidal growth pattern (Figure 4c and d). Interestingly, enlarged atypical Mott cells were also present that displayed kappa light chain restriction, consistent with a coexisting, unrelated plasma cell neoplasm. Precise classification of this case remained challenging in a patient who had received prior therapy, but considering bone marrow and splenic histology together, the findings favored reclassification as an lymphoplasmacytic lymphoma with relatively minor plasmacytic differentiation and a striking red pulp growth pattern in the spleen.

The final MYD88 mutated specimen was a mediastinal lymph node that showed a nodular infiltrate of small lymphocytes with pale cytoplasm and irregular nuclei admixed with IgM kappa monotypic plasmacytoid cells and showing prominent follicular colonization (Figure 5). No information was available in this case regarding bone marrow involvement or serum protein electrophoresis studies, but the morphologic findings remained most consistent with a nodal marginal zone lymphoma with plasmacytic differentiation.

Seven cases of small B-cell neoplasm with plasmacytic differentiation, not otherwise specified, were negative for the MYD88 mutation. Six of these showed similar features with a polymorphous infiltrate of small lymphocytes, plasma cells, occasional large transformed cells, and, in five of the six cases, prominent histiocytes (Figure 6). Immunohistochemistry showed three of these cases to utilize IgG heavy chains, and three to utilize IgM. Bone marrow involvement was documented in two out of four patients with available bone marrow biopsies.
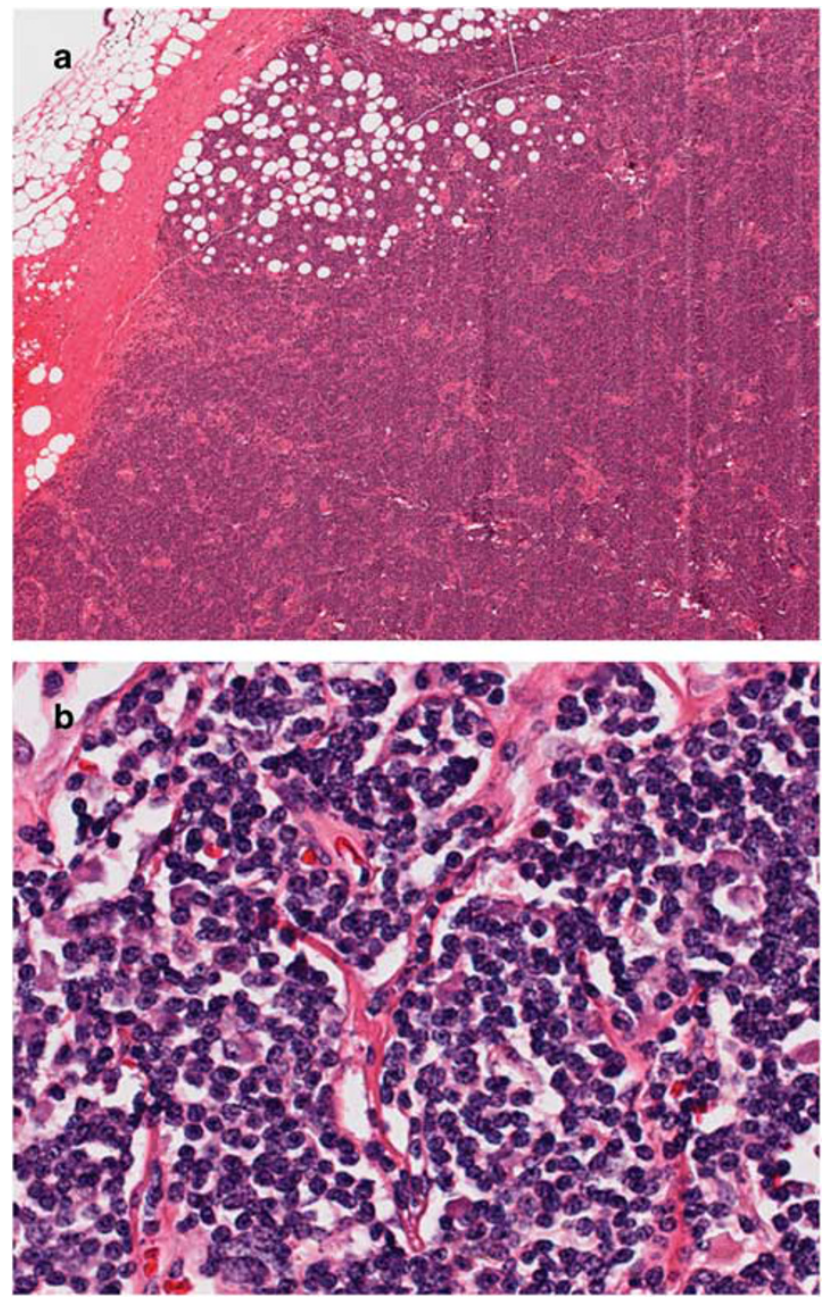

Figure 2 Three cases of small B-cell neoplasm with plasmacytic differentiation, not otherwise specified, with MYD88 mutations displayed diffuse architectural effacement (a) by a monomorphic lymphoplasmacytic infiltrate (b) cytologically similar to that seen in classic lymphoplasmacytic lymphoma.

The remaining case of small B-cell neoplasm with plasmacytic differentiation, not otherwise specified, displayed a diffuse infiltrate of small mature lymphocytes with clumped chromatin and a minor plasmacytic component. This case was negative for CD5 and positive for LEF1 and showed trisomy 12 by cytogenetics. This case therefore appeared consistent with atypical chronic lymphocytic leukemia/ small lymphocytic lymphoma with plasmacytic differentiation.

\section{Discussion}

Recently, next generation sequencing studies identified a highly recurrent point mutation, MYD88 L265P, in lymphoplasmacytic lymphoma. ${ }^{11}$ Mutations of MYD88, including the L265P point mutation, had been previously described in diffuse 

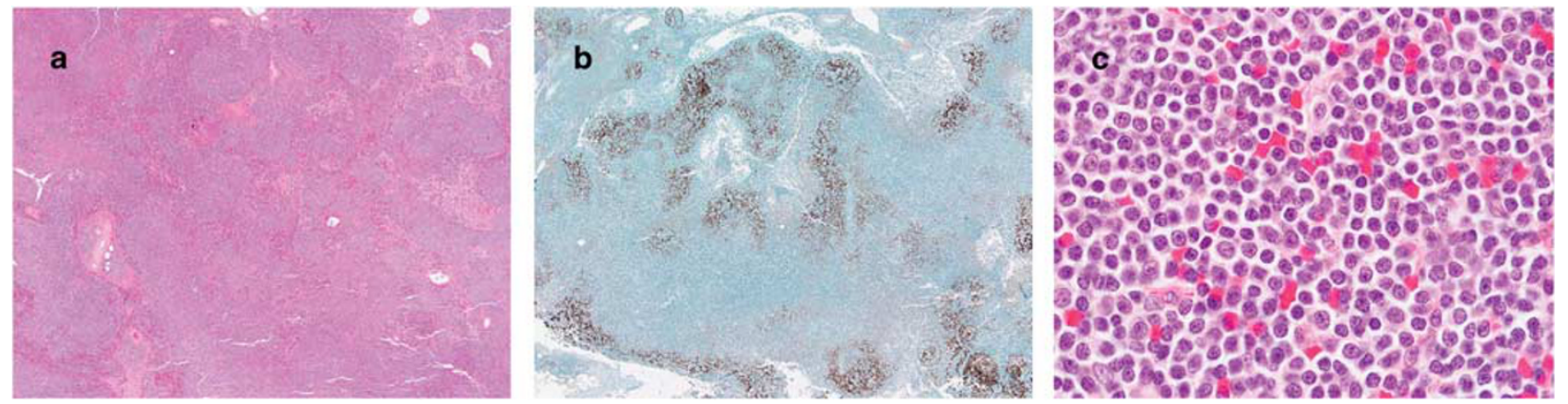

Figure 3 Four cases with MYD88 mutation (two originally classified as nodal marginal zone lymphoma with plasmacytic differentiation and two as small B-cell neoplasm with plasmacytic differentiation, not otherwise specified) showed similar features. Lymph node architecture was partially preserved with focally patent sinuses and vague to more prominent nodularity (a). Nodular areas corresponded to somewhat distorted follicular meshworks (b, CD21 IHC) suggestive of follicular colonization. The cellularity consisted of a monomorphic lymphoplasmacytic infiltrate similar to classic lymphoplasmacytic lymphoma cases (c).
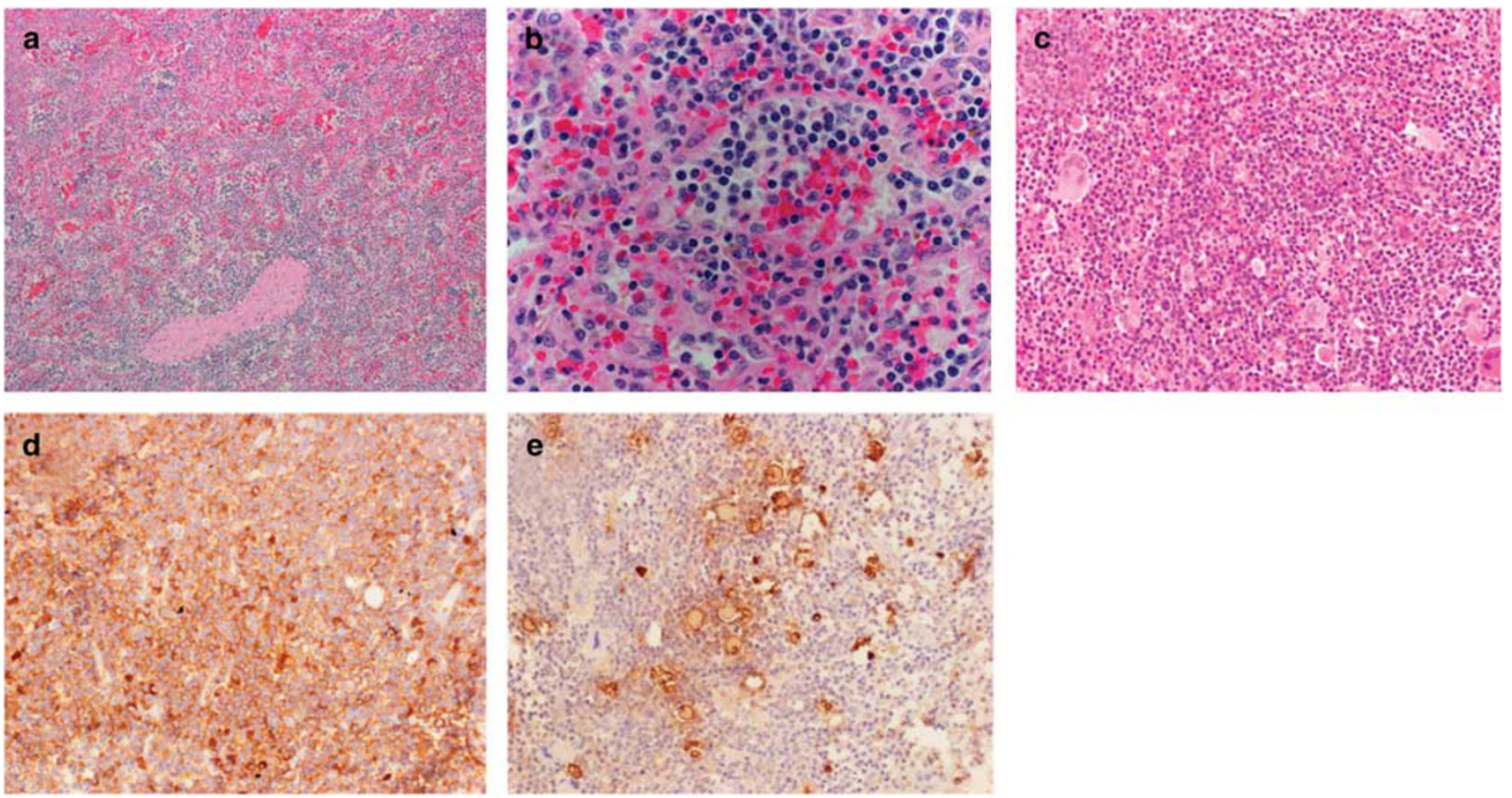

Figure 4 One case with MYD88 mutation was initially considered to represent an unclassifiable splenic lymphoma. This post-therapy splenectomy specimen displayed loss of the white pulp with a marked red pulp infiltrate (a) composed predominantly of small lymphocytes with rare plasmacytoid cells (b). Review of a previous bone marrow biopsy showed a diffuse infiltrate of lymphoplasmacytic cells (c) that expressed CD20 and IgM monotypic lambda (d, lambda IHC) although scattered kappa light chain restricted Mott cells were also present (e, kappa IHC).

large B-cell lymphomas and in $<10 \%$ of cases classified as extranodal marginal zone lymphoma. ${ }^{19,20}$ Subsequent studies from multiple institutions have confirmed MYD88 L265P to be present in the majority of lymphoplasmacytic lymphoma/ Waldenstrom's macroglobulinemia, with reported incidence from 67 to $93 \% .{ }^{12,15,21}$ The mutation has also been found in $44-87 \%$ of IgM monoclonal gammopathy of undetermined significance. ${ }^{12,15,22,23}$ In contrast, this abnormality has been found much less frequently in other small B-cell neoplasms, including $4-21 \%$ of cases diagnosed as splenic marginal zone lymphoma and $1-10 \%$ of chronic lymphocytic leukemia. ${ }^{11,12,15,23-26}$ The current study was designed to examine the morphologic correlates of MYD88 L265P in nodal and splenic small B-cell neoplasms with plasmacytic differentiation, and determine whether molecular studies for this mutation may assist in the diagnosis of lymphoplasmacytic lymphoma in routine practice. 
Overall, the MYD88 L265P mutation was found in $18 / 87$ cases $(18 \%)$. The presence of the mutation was significantly associated with an IgM
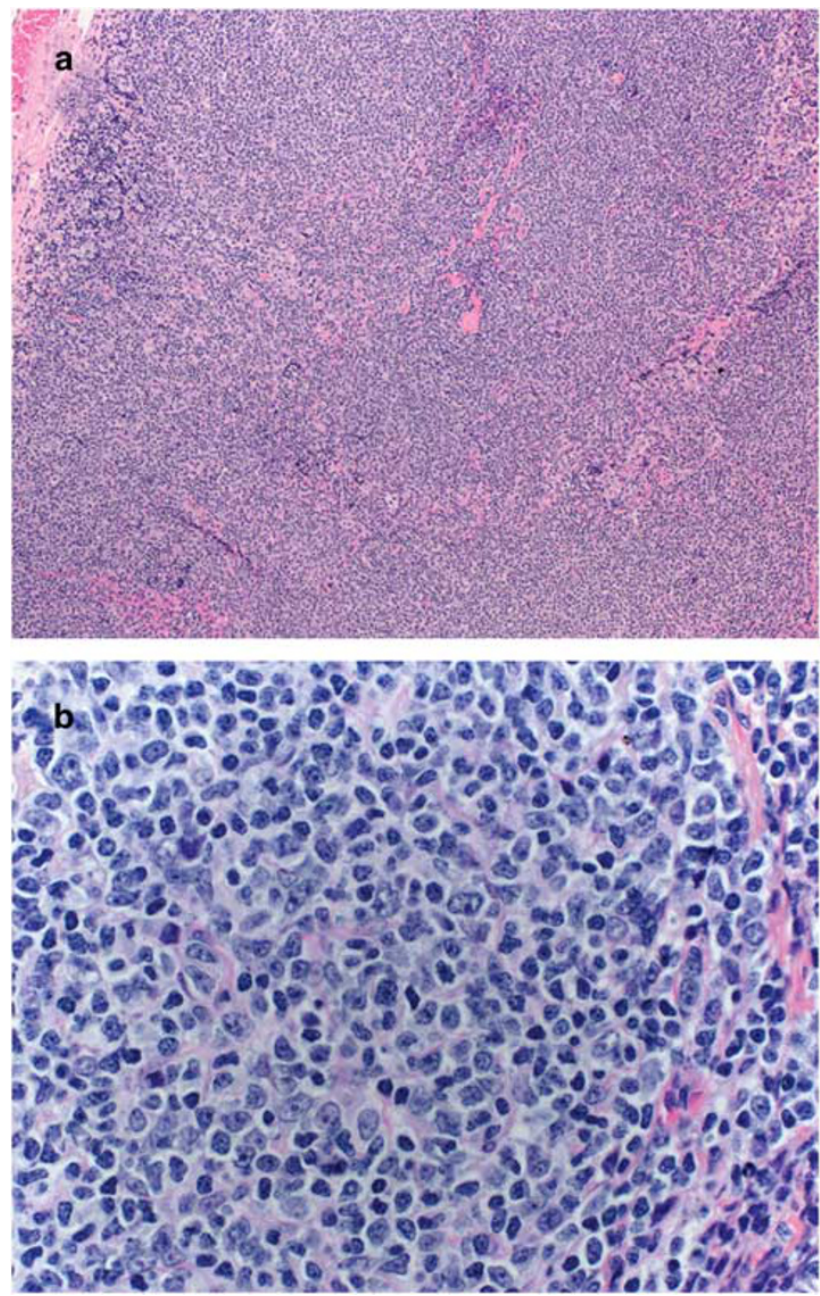

Figure 5 One MYD88 mutated nodal marginal zone lymphoma with plasmacytic differentiation showed architectural effacement by a vaguely nodular lymphoid proliferation (a) composed of small lymphocytes with irregular nuclear contours and scant amounts of pale cytoplasm (b). monoclonal protein $(P<0.001)$ and a trend towards bone marrow involvement $(P=0.09)$. These results are similar to prior reports showing that MYD88 mutation is associated with higher serum IgM levels in bone-marrow-based lymphoplasmacytic lymphoma and IgM monoclonal gammopathy of undetermined significance. ${ }^{12,15,21}$ Ho et $a l^{27}$ have also recently described MYD88 mutations to be associated with an IgM protein in nodal lymphomas as well.

For the purposes of this study, the 21 cases meeting the WHO definition of lymphoplasmacytic lymphoma were further subdivided in the initial morphologic review into classic lymphoplasmacytic lymphoma (based on the prototypical morphologic features) and small B-cell neoplasm with plasmacytic differentiation, not otherwise specified. Each of the nine cases of classic lymphoplasmacytic lymphoma was positive for the MYD88 L265P, and each was associated with bone marrow involvement and an IgM heavy chain. In light of these homogeneous clinicopathologic findings, it appears reasonable to suggest that nodal lymphomas with this constellation of morphologic features may be confidently diagnosed as lymphoplasmacytic lymphoma in routine practice without recourse to molecular studies for MYD88 mutations.

In contrast, the cases categorized as small B-cell neoplasm with plasmacytic differentiation, not otherwise specified, are clearly more heterogeneous. Overall, five cases of small B-cell neoplasm with plasmacytic differentiation, not otherwise specified, were positive for the MYD88 L265P mutation. Three of these cases were cytologically similar to classic lymphoplasmacytic lymphoma, but showed diffuse architectural effacement and involvement of the perinodal soft tissues. Each of these three cases was also associated with bone marrow involvement and an IgM heavy chain. Upon re-review, these three cases therefore appeared to represent nodal lymphoplasmacytic lymphoma with more diffuse architectural effacement than typically described in prototypical lymphoplasmacytic lymphoma. Somewhat more challenging to categorize are four cases
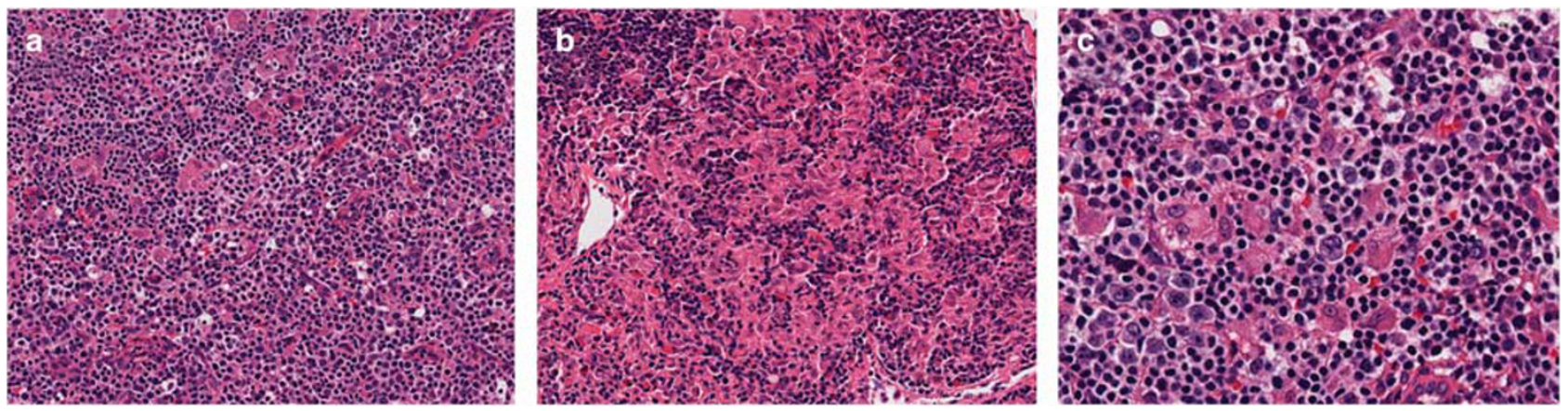

Figure 6 Six cases classified as small B-cell neoplasm with plasmacytic differentiation, not otherwise specified lacked MYD88 mutations and showed similar features with complete architectural effacement (a) by a polymorphic infiltrate of small lymphocytes, plasmacytoid cells, and plasma cells with occasional large transformed cells and varyingly prominent epithelioid histiocytes (b, c). 
with MYD88 L265P mutations, two originally considered small B-cell neoplasm with plasmacytic differentiation, not otherwise specified, and two originally classified as nodal marginal zone lymphomas with plasmacytic differentiation. These cases each showed similar findings with partially preserved architecture characterized by at least focally patent sinuses, a vaguely nodular growth pattern, variably prominent follicular colonization (more readily identified in the cases initially classified as marginal zone lymphoma), and a lymphoplasmacytic infiltrate that was largely similar to that seen in classic lymphoplasmacytic lymphoma and only focal areas where the cells were monocytoid. Overall, the clinicopathologic features in these four cases favored classification as lymphoplasmacytic lymphoma with a vaguely nodular architecture and variably prominent follicular colonization. Although follicular colonization is frequently seen in marginal zone lymphoma, this phenomenon may also be seen in other B-cell lymphomas, including follicular lymphoma ${ }^{28-31}$ and mantle cell lymphoma. ${ }^{32}$ In the 2009 Society for Hematopathology/European Association of Hematopathology workshop, cases of putative lymphoplasmacytic lymphoma with follicular colonization were accepted as bona fide lymphoplasmacytic lymphoma by some, but not all, members of the expert review panel. ${ }^{4}$ The presence of MYD88 L265P mutations in such cases, as observed in this study, also suggests that such cases may represent bona fide lymphoplasmacytic lymphoma. Finally, one case initially categorized as an unclassifiable splenic B-cell lymphoma also was positive for the MYD88 L265P mutation. This case displayed a remarkable red pulp growth pattern in the spleen, suggesting a possible diagnosis of diffuse red pulp small B-cell lymphoma, a provisional entity in the 2008 WHO classification where the cells are described as having plasmacytoid features. ${ }^{33-35}$ Subsequent examination of earlier bone marrow biopsies, however, showed no sign of the intrasinusoidal growth pattern characteristic of this entity, but rather a diffuse lymphoplasmacytic infiltrate that would be consistent with a diagnosis of lymphoplasmacytic lymphoma. Although there are little data in the recent literature regarding the splenic histology of lymphoplasmacytic lymphoma as defined by 2008 WHO criteria, the older literature does describe cases of putative lymphoplasmacytic lymphoma with a predominantly red pulp pattern of involvement. ${ }^{36,37}$ It is also unclear whether prior treatment might have also contributed to the red pulp pattern observed in the splenectomy specimen. This case remains challenging to classify, but the overall features suggest this case might also be best considered lymphoplasmacytic lymphoma.

These results indicate that although cases of lymphoplasmacytic lymphoma showing architectural preservation with patent sinuses and a diffuse growth pattern may be the easiest subset of lymphoplasmacytic lymphoma to recognize, the presence of complete architectural effacement, perinodal soft tissue involvement, a vaguely nodular growth pattern, or follicular colonization should not exclude a diagnosis of lymphoplasmacytic lymphoma. It is in these histologically more challenging cases that MYD88 L265P mutation analysis may be most helpful in refining a differential diagnosis. The unifying feature in all of these cases is the relatively monomorphic cytologic appearance supporting the diagnosis of a MYD88 mutated lymphoplasmacytic lymphoma.

The previously published studies of MYD88 mutation status in lymphoplasmacytic lymphoma have largely focused on IgM- associated lymphoplasmacytic lymphoma (ie, Waldenstrom's macroglobulinemia). Only a small number of non-IgMassociated lymphoplasmacytic lymphomas have been examined with varying results. At least some cases of IgG- and IgA-associated lymphoplasmacytic lymphoma ${ }^{11,14,38}$ have been reported to be positive for MYD88 L265P, while other investigators have reported non-IgM lymphoplasmacytic lymphoma to lack the mutation. ${ }^{12,39}$ In the current study, we identified one case of MYD88 mutated lymphoplasmacytic lymphoma (with follicular colonization) that was associated with an IgG paraprotein by immunofixation. Examination by immunohistochemistry, however, showed the clonal plasma cells to be positive for IgM, and review of the electronic medical record showed an earlier immunofixation study with both IgM and IgG monoclonal proteins. These results emphasize that both serum immunofixation and immunohistochemistry data should be considered in analysis of what appears to be a non-IgM lymphoplasmacytic lymphoma.

Following the second round of morphologic review, only a single MYD88 L265P mutated case was felt to be incompatible with a diagnosis of lymphoplasmacytic lymphoma. This case displayed a prominently nodular growth pattern and cytology that appeared much more consistent with a diagnosis of nodal marginal zone lymphoma with plasmacytic differentiation. MYD88 L265P has also been described in a small percentage of chronic lymphocytic leukemia/small lymphocytic lymphoma, ${ }^{15,21,25,26,40,41}$ emphasizing that MYD88 mutation status alone clearly cannot be used to define lymphoplasmacytic lymphoma. The MYD88 L265P abnormality has also been reported in a small percentage of cases diagnosed as extranodal marginal zone lymphoma, ${ }^{19,20}$ although distinction between extranodal marginal zone lymphoma with plasmacytic differentiation and an extranodal lymphoplasmacytic lymphoma may be somewhat arbitrary, and the prior studies included insufficient clinical or histologic information to completely exclude extranodal lymphoplasmacytic lymphoma. Furthermore, lymphoplasmacytic lymphoma is well documented to involved mucosa-associated 
lymphoid tissue sites in a small number of cases. ${ }^{2,4,42}$ The lack of specificity of the MYD88 L265P mutation for lymphoplasmacytic lymphoma is clearly evident from its presence in up to $30 \%$ of activated B-cell type diffuse large B-cell lymphoma and in an even higher proportion of primary cutaneous diffuse large B-cell lymphoma, leg type. ${ }^{43-47}$ Additional studies of extranodal marginal zone lymphomas with plasmacytic differentiation may be helpful to determine the utility of MYD88 mutation analysis in extranodal, extramedullary biopsy specimens.

The differential diagnosis of lymphoplasmacytic lymphoma vs splenic marginal zone lymphoma is particularly problematic. Splenic marginal zone lymphoma often shows plasmacytic differentiation, and when associated with a monoclonal protein, it is usually of IgM type. ${ }^{48-52}$ Like lymphoplasmacytic lymphoma, splenic marginal zone lymphoma generally shows a nonspecific CD5-negative, CD10-negative phenotype. Splenic marginal zone lymphoma and lymphoplasmacytic lymphoma each show a similar demographic distribution and the initial clinical presentation may be similar. Several prior studies have therefore examined the incidence of MYD88 L265P in splenic marginal zone lymphoma to determine the utility of this marker in differential diagnosis, with positive results reported from 4 to $21 \%$ of cases. ${ }^{11,12,14,15,22}$ Most of the cases reported to date, however, have been diagnosed from peripheral blood and bone marrow alone. Moreover, it is unclear how many of these splenic marginal zone lymphomas may show evidence of plasmacytic differentiation such that the differential diagnosis would include lymphoplasmacytic lymphoma. One prior study which was restricted to splenic marginal zone lymphoma confirmed by splenic histology reported MYD88 L265P in $2 / 53(4 \%)$ cases. ${ }^{22}$ In the current study, we included only splenectomy-defined splenic marginal zone lymphoma cases, and included cases with and without plasmacytic differentiation. Notably, none of the 44 cases of splenic marginal zone lymphoma showed evidence of MYD88 mutation. Our findings indicate that the MYD88 mutation is, at best, rare in splenectomy-defined splenic marginal zone lymphoma, even in those with plasmacytic differentiation and an IgM protein, and suggest that PCR for the MYD88 L265P mutation can be very useful in distinguishing between lymphoplasmacytic lymphoma and splenic marginal zone lymphoma, even if not absolute.

The small B-cell neoplasms associated with cold agglutinin disease have also been challenging to classify. Many such cases have generally been accepted as lymphoplasmacytic lymphoma in the prior literature. ${ }^{53,54}$ Interestingly, one case in the current series represented a splenic- and bone-marrow-based small B-cell neoplasm with plasmacytic differentiation associated with cold agglutinin disease. For purposes of this study, this lymphoma was placed into the category of unclassifiable splenic B-cell lymphoma rather than lymphoplasmacytic lymphoma because although it contained white pulp aggregates of small lymphocytes and plasma cells suggestive of lymphoplasmacytic lymphoma, it also displayed a prominent histiocytic and overtly granulomatous infiltrate that was considered unusual for typical lymphoplasmacytic lymphoma. This case lacked the MYD88 L265P mutation. The findings are similar to those of a recent study showing that cold agglutinin associated B-cell lymphoproliferative disorders lack the MYD88 L265P mutation. ${ }^{55}$ These results suggest that lymphomas associated with cold agglutinin disease arise through a different pathogenetic mechanism than typical lymphoplasmacytic lymphoma, and that these may be considered to represent distinct entities. However, this must be further clarified as cold agglutinins have been reported in one study in $27 \%$ of MYD88 L2565P mutated lymphoplasmacytic lymphoma. ${ }^{14}$

This study has also identified a subset of cases that show similar morphologic findings, but lack the MYD88 L265P mutation. These cases, which met the diagnosis-of-exclusion criteria for lymphoplasmacytic lymphoma as currently defined, showed diffuse architectural effacement, a polymorphous infiltrate of small lymphocytes, plasma cells, and scattered large transformed cells, and were associated with a histiocyte-rich background. Interestingly, these cases are morphologically very similar to a subset of cases of gamma heavy chain disease, which we described in a prior study, ${ }^{56}$ and have recently shown to also lack the MYD88 L265P mutation. ${ }^{57}$ These cases, which we have tentatively designated polymorphous/histiocyte-rich B-cell lymphoma with plasmacytic differentiation must be further studied to determine whether they may represent a form of marginal zone lymphoma or whether they might be better considered to represent a distinct neoplasm.

In conclusion, the findings in this study help clarify the morphologic spectrum of nodal lymphoplasmacytic lymphoma. In addition to the cases showing the classic histologic features of nodal lymphoplasmacytic lymphoma, which are uniformly positive for MYD88 L265P, these findings indicate that nodal lymphoplasmacytic lymphoma may sometimes show features previously considered atypical of lymphoplasmacytic lymphoma including diffuse architectural effacement, perinodal soft tissue involvement, a vaguely nodular growth pattern, and variably prominent follicular colonization. Our results, together with the prior literature, emphasize that MYD88 L265P mutation detection can be very helpful in the differential diagnosis of lymphoplasmacytic lymphoma vs splenic marginal zone lymphoma, but that occasional non-lymphoplasmacytic lymphoma small B-cell neoplasms, such as nodal marginal zone lymphoma or chronic lymphocytic leukemia/small lymphocytic 
lymphoma may also be associated with this mutation. Lastly, this study also identifies a cohort of cases (polymorphous/histiocyte-rich B-cell lymphoma with plasmacytic differentiation) that is morphologically distinct from typical lymphoplasmacytic lymphoma, is less likely to be associated with IgM heavy chains and bone marrow involvement, lacks the MYD88 L265P mutation, and should no longer be considered to represent lymphoplasmacytic lymphoma.

\section{Disclosure/conflict of interest}

The authors declare no conflict of interest.

\section{References}

1 Lin P, Medeiros LJ. Lymphoplasmacytic lymphoma/ waldenstrom macroglobulinemia: an evolving concept. Adv Anat Pathol 2005;12:246-255.

2 Swerdlow SH, Berger F, Pileri SA, et al. Lymphoplasmacytic Lymphoma, In: Swerdlow SH, Campo E, Harris NL, et al. (eds), WHO Classification of Tumours of Haematopoietic and Lymphoid Tissues. IARC Press: Lyon; 2008, pp 194-195.

3 Cook JR. Nodal and leukemic small B-cell neoplasms. Mod Pathol 2013;26(Suppl 1):S15-S28.

4 Lin P, Molina TJ, Cook JR, et al. Lymphoplasmacytic lymphoma and other non-marginal zone lymphomas with plasmacytic differentiation. Am J Clin Pathol 2011;136:195-210.

5 Bennett M, Farrer-Brown G, Henry K, et al. Classification of non-Hodgkin's lymphomas. Lancet 1974;304: 405-408.

6 Lukes RJ, Collins RD. Immunologic characterization of human malignant lymphomas. Cancer 1974;34(suppl): 1488-1503.

7 Andriko JA, Swerdlow SH, Aguilera NI, et al. Is lymphoplasmacytic lymphoma/immunocytoma a distinct entity? A clinicopathologic study of 20 cases. Am J Surg Pathol 2001;25:742-751.

8 Sargent RL, Cook JR, Aguilera NI, et al. Fluorescence immunophenotypic and interphase cytogenetic characterization of nodal lymphoplasmacytic lymphoma. Am J Surg Pathol 2008;32:1643-1653.

9 Cook JR, Aguilera NI, Reshmi S, et al. Deletion 6q is not a characteristic marker of nodal lymphoplasmacytic lymphoma. Cancer Genet Cytogenet 2005;162:85-88.

10 Cook JR, Aguilera NI, Reshmi-Skarja S, et al. Lack of PAX5 rearrangements in lymphoplasmacytic lymphomas: reassessing the reported association with $\mathrm{t}(9 ; 14)$. Hum Pathol 2004;35:447-454.

11 Treon SP, Xu L, Yang G, et al. MYD88 L265P Somatic Mutation in Waldenström's Macroglobulinemia. N Engl J Med 2012;367:826-833.

12 Jiménez C, Sebastián E, Chillón MC, et al. MYD88 L265P is a marker highly characteristic of, but not restricted to, Waldenström's macroglobulinemia. Leukemia 2013;27:1722-1728.

13 Treon SP, Hunter ZR. A new era for Waldenstrom macroglobulinemia: MYD88 L265P. Blood 2013;121: 4434-4436.
14 Poulain S, Roumier C, Decambron A, et al. MYD88 L265P mutation in Waldenstrom macroglobulinemia. Blood 2013;121:4504-4511.

$15 \mathrm{Xu} \mathrm{L}$, Hunter ZR, Yang G, et al. MYD88 L265P in Waldenström macroglobulinemia, immunoglobulin $\mathrm{M}$ monoclonal gammopathy, and other B-cell lymphoproliferative disorders using conventional and quantitative allele-specific polymerase chain reaction. Blood 2013;121:2051-2058.

16 Mori N, Ohwashi M, Yoshinaga K, et al. L265P mutation of the MYD88 gene Is frequent in Waldenström's macroglobulinemia and its absence in myeloma. PLoS One 2013;8:e80088.

17 Ondrejka SL, Lin JJ, Warden DW, et al. MYD88 L265P somatic mutation: its usefulness in the differential diagnosis of bone marrow involvement by B-cell lymphoproliferative disorders. Am J Clin Pathol 2013;140:387-394.

18 Swerdlow SH, Campo E, Harris NL, et al. WHO Classification of Tumours of Haematopoietic and Lymphoid Tissues. IARC Press: Lyon; 2008, pp 179-269.

19 Ngo VN, Young RM, Schmitz R, et al. Oncogenically active MYD88 mutations in human lymphoma. Nature 2011;470:115-119.

20 Li Z-M, Rinaldi A, Cavalli A, et al. MYD88 somatic mutations in MALT lymphomas. Br J Haematol 2012;158:662-664.

21 Varettoni M, Arcaini L, Zibellini S, et al. Prevalence and clinical significance of the MYD88 (L265P) somatic mutation in Waldenstrom's macroglobulinemia and related lymphoid neoplasms. Blood 2013; 121:2522-2528.

22 Gachard N, Parrens M, Soubeyran I, et al. IGHV gene features and MYD88 L265P mutation separate the three marginal zone lymphoma entities and Waldenström macroglobulinemia/lymphoplasmacytic lymphomas. Leukemia 2013;27:183-189.

23 Landgren O, Staudt L. MYD88 L265P somatic mutation in IgM MGUS. N Engl J Med 2012;367:2255-2256.

24 Wang L, Lawrence MS, Wan Y, et al. SF3B1 and other novel cancer genes in chronic lymphocytic leukemia. N Engl J Med 2011;365:2497-2506.

25 Martínez-Trillos A, Pinyol M, Navarro A, et al. Mutations in the Toll-like receptor/MYD88 pathway in chronic lymphocytic leukemia identify a subset of young patients with favorable outcome. Blood 2014;123:3790-3796.

26 Puente XS, Pinyol M, Quesada V, et al. Whole-genome sequencing identifies recurrent mutations in chronic lymphocytic leukaemia. Nature 2011;475:101-105.

27 Ho Y, Xu L, Treon SP, et al. MYD88 L265P mutation analysis aids in classifying nodal small B-cell lymphomas. Mod Pathol 2014;27:351A.

28 Carbone A, Gloghini A, Santoro A. In situ follicular lymphoma: pathologic characteristics and diagnostic features. Hematol Oncol 2012;30:1-7.

29 Jegalian AG, Eberle FC, Pack SD, et al. Follicular lymphoma in situ: clinical implications and comparisons with partial involvement by follicular lymphoma. Blood 2011;118:2976-2984.

30 Mamessier E, Broussais-Guillaumot F, Chetaille B, et al. Nature and importance of follicular lymphoma precursors. Haematologica 2014;99:802-810.

$31 \mathrm{Xu}$ Y, McKenna RW, Kroft SH. Assessment of CD10 in the diagnosis of small B-cell lymphomas: a multiparameter flow cytometric study. Am J Clin Pathol 2002;117:291-300. 
32 Yatabe Y, Suzuki R, Matsuno Y, et al. Morphological spectrum of cyclin D1-positive mantle cell lymphoma: study of 168 cases. Pathol Int 2001;51: 747-761.

33 Traverse-Glehen A, Baseggio L, Bauchu EC, et al. Splenic red pulp lymphoma with numerous basophilic villous lymphocytes: a distinct clinicopathologic and molecular entity? Blood 2008;111:2253-2260.

34 Mollejo M, Algara P, Mateo MS, et al. Splenic small B-cell lymphoma with predominant red pulp involvement: a diffuse variant of splenic marginal zone lymphoma? Histopathology 2002;40:22-30.

35 Piris MA, Foucar K, Mollejo M, et al. Splenic B-cell lymphoma/leukemia, unclassifiable, In: Swerdlow SH, Campo E, Harris NL, et al. (eds), WHO Classification of Tumours of Haematopoietic and Lymphoid Tissues. IARC Press: Lyon; 2008, pp 191-193.

36 Arber DA, Rappaport H, Weiss LM. Non-Hodgkin's lymphoproliferative disorders involving the spleen. Mod Pathol. 1997;10:18-32.

37 Lin P, Bueso-Ramos C, Wilson CS, et al. Waldenstrom macroglobulinemia involving extramedullary sites: morphologic and immunophenotypic findings in 44 patients. Am J Surg Pathol. 2003;27:1104-1113.

38 Anelli L, Zagaria A, Minervini A, et al. IgG-lymphoplasmacytic lymphoma following polycythemia vera: JAK2 V617F and MYD88 L265P mutations separated in the same house. Ann Hematol 2014;3:1-9.

39 Manasanch EE, Braylan R, Stetler-Stevenson M, et al. Lack of MYD88 L265P in non-immunoglobulin M lymphoplasmacytic lymphoma. Leuk Lymphoma 2013;55:1402-1403.

40 Cortese D, Sutton L-A, Cahill N, et al. On the way towards a 'CLL prognostic index': focus on TP53, BIRC3, SF3B1, NOTCH1 and MYD88 in a populationbased cohort. Leukemia 2014;28:710-713.

41 Jeromin S, Weissmann S, Haferlach C, et al. SF3B1 mutations correlated to cytogenetics and mutations in NOTCH1, FBXW7, MYD88, XPO1 and TP53 in 1160 untreated CLL patients. Leukemia 2014;28: 108-117.

42 Kyrtsonis MC, Vassilakopoulos TP, Angelopoulou MK, et al. Waldenström's macroglobulinemia: clinical course and prognostic factors in 60 patients. Experience from a single hematology unit. Ann Hematol 2001;80:722-727.

43 Choi J-W, Kim Y, Lee J-H, et al. MYD88 expression and L265P mutation in diffuse large B-cell lymphoma. Hum Pathol 2013;44:1375-1381.

44 Kraan W, Horlings HM, van Keimpema M, et al. High prevalence of oncogenic MYD88 and CD79B mutations in diffuse large B-cell lymphomas presenting at immune-privileged sites. Blood Cancer J 2013;3:e139.

45 Pham-Ledard A, Prochazkova-Carlotti M, Andrique L, et al. Multiple genetic alterations in primary cutaneous large B-cell lymphoma, leg type support a common lymphomagenesis with activated B-cell-like diffuse large B-cell lymphoma. Mod Pathol 2014;27:402-411.

46 Kraan W, van Keimpema M, Horlings HM, et al. High prevalence of oncogenic MYD88 and CD79B mutations in primary testicular diffuse large B-cell lymphoma. Leukemia 2014;28:719-720.

$47 \mathrm{Kim} \mathrm{Y}$, Ju H, Kim DH, et al. CD79B and MYD88 mutations in diffuse large B-cell lymphoma. Hum Pathol 2014;45:556-564.

48 Molina TJ, Lin P, Swerdlow SH, et al. Marginal zone lymphomas with plasmacytic differentiation and related disorders. Am J Clin Pathol 2011;136:211-225.

49 Oscier D, Owen R, Johnson S. Splenic marginal zone lymphoma. Blood Rev 2005;19:39-51.

50 Van Huyen JP, Molina T, Delmer A, et al. Splenic marginal zone lymphoma with or without plasmacytic differentiation. Am J Surg Pathol 2000;24:1581-1592.

51 Dufresne SD, Felgar RE, Sargent RL, et al. Defining the borders of splenic marginal zone lymphoma: a multiparameter study. Hum Pathol 2010;41:540-551.

52 Isaacson PG, Piris MA, Berger F, et al. Splenic B-cell marginal zone lymphoma, In: Swerdlow SH, Campo E, Harris NL, et al. (eds), WHO Classification of Tumours of Haematopoietic and Lymphoid Tissues. IARC Press: Lyon; 2008, pp 185-187.

53 Berentsen S, Beiske K, Tjønnfjord GE. Primary chronic cold agglutinin disease: an update on pathogenesis, clinical features and therapy. Hematology 2007;12: 361-370.

54 Berentsen S, Ulvestad E, Langholm R, et al. Primary chronic cold agglutinin disease: a population based clinical study of 86 patients. Haematologica 2006; 91:460-466.

55 Randen U, Trøen G, Tierens A, et al. Primary cold agglutinin-associated lymphoproliferative disease: a B-cell lymphoma of the bone marrow distinct from lymphoplasmacytic lymphoma. Haematologica 2014; 99:497-504.

56 Bieliauskas S, Tubbs RR, Bacon CM, et al. Gamma heavy-chain disease: defining the spectrum of associated lymphoproliferative disorders through analysis of 13 cases. Am J Surg Pathol 2012;36:534-543.

57 Hamadeh F, MacNamara S, Bacon CM, et al. Gamma heavy chain disease lacks the MYD88 L265P mutation associated with lymphoplasmacytic lymphoma. Haematologica 23 May 2014; e-pub ahead of print. 